\title{
Relaxed Halpern-type iteration method for countable families of totally quasi- $\phi$-asymptotically nonexpansive mappings
}

Wei-Qi Deng*

"Correspondence:

dwq1273@126.com

College of Statistics and

Mathematics, Yunnan University of

Finance and Economics, Kunming,

Yunnan 650221, P.R. China

\begin{abstract}
Based on an original idea, namely, a specific way of choosing the indexes of involved mappings, we propose a relaxed Halpern-type iterative algorithm for approximating some common fixed point of a kind of nonlinear mappings and obtain a strong convergence theorem under suitable conditions. Since the involved mappings need no assumption of being uniformly totally quasi- $\phi$-asymptotically nonexpansive, and there is no need to compute projections onto intersections of countably many closed and convex sets, the results improve those of the authors with related interest.
\end{abstract}

MSC: 47H09; 47H10;47J25

Keywords: Halpern-type iteration; totally quasi- $\phi$-asymptotically nonexpansive mappings; generalized projection

\section{Introduction}

Throughout this paper, we assume that $E$ is a real Banach space with its dual $E^{*}, C$ is a nonempty closed convex subset of $E$, and $J: E \rightarrow 2^{E^{*}}$ is the normalized duality mapping defined by

$$
J x=\left\{f \in E^{*}:\langle x, f\rangle=\|x\|^{2}=\|f\|^{2}\right\}, \quad \forall x \in E .
$$

In the sequel, we use $F(T)$ to denote the set of fixed points of a mapping $T$.

Definition 1.1 [1] A mapping $T: C \rightarrow C$ is said to be totally quasi- $\phi$-asymptotically nonexpansive if $F(T) \neq \emptyset$ and there exist nonnegative real sequences $\left\{v_{n}\right\},\left\{\mu_{n}\right\}$ with $v_{n}, \mu_{n} \rightarrow 0$ $($ as $n \rightarrow \infty)$ and a strictly increasing continuous function $\zeta: \mathbb{R}^{+} \rightarrow \mathbb{R}^{+}$with $\zeta(0)=0$ such that

$$
\phi\left(p, T^{n} x\right) \leq \phi(p, x)+v_{n} \zeta(\phi(p, x))+\mu_{n}, \quad \forall n \geq 1, x \in C, p \in F(T)
$$

where $\phi: E \times E \rightarrow \mathbb{R}^{+}$denotes the Lyapunov functional defined by

$$
\phi(x, y)=\|x\|^{2}-2\langle x, J y\rangle+\|y\|^{2}, \quad \forall x, y \in E .
$$

@ 2013 Deng; licensee Springer. This is an Open Access article distributed under the terms of the Creative Commons Attribution License (http://creativecommons.org/licenses/by/2.0), which permits unrestricted use, distribution, and reproduction in any medium, provided the original work is properly cited. 
It is obvious from the definition of $\phi$ that

$$
(\|x\|-\|y\|)^{2} \leq \phi(x, y) \leq(\|x\|+\|y\|)^{2} .
$$

Definition 1.2 [1] (1) A countable family of mappings $\left\{T_{i}\right\}: C \rightarrow C$ is said to be totally uniformly quasi- $\phi$-asymptotically nonexpansive if $F:=\bigcap_{i=1}^{\infty} F\left(T_{i}\right) \neq \emptyset$ and there exist nonnegative real sequences $\left\{v_{n}\right\},\left\{\mu_{n}\right\}$ with $v_{n}, \mu_{n} \rightarrow 0$ (as $\left.n \rightarrow \infty\right)$ and a strictly increasing continuous function $\zeta: \mathbb{R}^{+} \rightarrow \mathbb{R}^{+}$with $\zeta(0)=0$ such that

$$
\phi\left(p, T_{i}^{n} x\right) \leq \phi(p, x)+v_{n} \zeta(\phi(p, x))+\mu_{n}, \quad \forall n \geq 1, i \geq 1, x \in C, p \in F(T)
$$

(2) A mapping $T: C \rightarrow C$ is said to be uniformly L-Lipschitz continuous if there exists a constant $L>0$ such that

$$
\left\|T^{n} x-T^{n} y\right\| \leq L\|x-y\|, \quad \forall n \geq 1, x, y \in C .
$$

In 2012, Chang et al. [1] used the following modified Halpern-type iteration algorithm for totally quasi- $\phi$-asymptotically nonexpansive mappings to have the strong convergence under a limit condition only in the framework of Banach spaces.

$$
\left\{\begin{array}{l}
x_{1} \in C ; \quad C_{1}=C, \\
y_{n, m}=J^{-1}\left[\alpha_{n} J x_{1}+\left(1-\alpha_{n}\right) J T_{m}^{n} x_{n}\right], \quad m \geq 1, \\
C_{n+1}=\left\{z \in C_{n}: \sup _{m \geq 1} \phi\left(z, y_{n, m}\right) \leq \alpha_{n} \phi\left(z, x_{1}\right)+\left(1-\alpha_{n}\right) \phi\left(z, x_{n}\right)+\xi_{n}\right\} \\
x_{n+1}=\Pi_{C_{n+1}} x_{1}, \quad \forall n \geq 1,
\end{array}\right.
$$

where $\left\{T_{i}\right\}: C \rightarrow C$ is a countable family of closed and uniformly totally quasi- $\phi$ asymptotically nonexpansive mappings; and $\xi_{n}=v_{n} \sup _{p \in F} \zeta\left(\phi\left(p, x_{n}\right)\right)+\mu_{n}, \Pi_{C_{n+1}}$ is the generalized projection (see (2.1)) of $E$ onto $C_{n+1}$. Their results extended and improved the corresponding results of Qin et al. [2, 3], Wang et al. [4], Martinez-Yanes and Xu [5] and others.

However, it is obviously a very strong condition that the involved mappings are assumed to be uniformly totally quasi- $\phi$-asymptotically nonexpansive. Additionally, the assumption conditions imposed on all $C_{n}(n \geq 2)$ are not weak at all since each one is in fact an intersection of countably many sets. This fact makes the projection very hard to compute, and therefore the method proposed in their paper does not seem to be valuable in practice.

Inspired and motivated by the studies mentioned above, in this article, we introduce a relaxed iterative algorithm for approximating some common fixed point of a countable family of totally quasi- $\phi$-asymptotically nonexpansive mappings and obtain a strong convergence theorem.

\section{Preliminaries}

Following Alber [6], the generalized projection $\Pi_{C}: E \rightarrow C$ is defined by

$$
\Pi_{C}=\arg \inf _{y \in C} \phi(y, x), \quad \forall x \in E .
$$


Lemma 2.1 [6] Let E be a smooth, strictly convex and reflexive Banach space, and let $C$ be a nonempty closed convex subset of $E$. Then the following conclusions hold:

(1) $\phi\left(x, \Pi_{C} y\right)+\phi\left(\Pi_{C} y, y\right) \leq \phi(x, y)$ for all $x \in C$ and $y \in E$;

(2) If $x \in E$ and $z \in C$, then $z=\Pi_{C} x \Leftrightarrow\langle z-y, J x-J z\rangle \geq 0, \forall y \in C$;

(3) For $x, y \in E, \phi(x, y)=0$ if and only if $x=y$.

Remark 2.2 The following basic properties for a Banach space $E$ can be found in Cioranescu [7].

(i) If $E$ is uniformly smooth, then $J$ is uniformly continuous on each bounded subset of $E$;

(ii) If $E$ is reflexive and strictly convex, then $J^{-1}$ is norm-weak-continuous;

(iii) If $E$ is a smooth, strictly convex and reflexive Banach space, then the normalized duality mapping $J: E \rightarrow 2^{E^{*}}$ is single-valued, one-to-one and onto;

(iv) A Banach space $E$ is uniformly smooth if and only if $E^{*}$ is uniformly convex;

(v) Each uniformly convex Banach space $E$ has the Kadec-Klee property, i.e., for any sequence $\left\{x_{n}\right\} \subset E$, if $x_{n} \rightarrow x \in E$ and $\left\|x_{n}\right\| \rightarrow\|x\|$, then $x_{n} \rightarrow x$ as $n \rightarrow \infty$.

Lemma 2.3 [1] Let E be a real uniformly smooth and strictly convex Banach space with the Kadec-Klee property, and let $C$ be a nonempty closed convex subset of E. Let $\left\{x_{n}\right\}$ and $\left\{y_{n}\right\}$ be two sequences in $C$ such that $x_{n} \rightarrow p$ and $\phi\left(x_{n}, y_{n}\right) \rightarrow 0$, where $\phi$ is the function defined by (1.2), then $y_{n} \rightarrow p$.

Lemma 2.4 [1] Let $E$ and $C$ be the same as in Lemma 2.3. Let $T: C \rightarrow C$ be a closed and totally quasi- $\phi$-asymptotically nonexpansive mapping with nonnegative real sequences $\left\{v_{n}\right\}$, $\left\{\mu_{n}\right\}$ and a strictly increasing continuous function $\zeta: \mathbb{R}^{+} \rightarrow \mathbb{R}^{+}$such that $v_{n}, \mu_{n} \rightarrow 0$ and $\zeta(0)=0$. If $\mu_{1}=0$, then the fixed point set $F(T)$ of $T$ is a closed and convex subset of $C$.

Lemma 2.5 [8] The unique solutions to the positive integer equation

$$
n=i+\frac{(m-1) m}{2}, \quad m \geq i, n=1,2,3, \ldots
$$

are

$$
i=n-\frac{(m-1) m}{2}, \quad m=-\left[\frac{1}{2}-\sqrt{2 n+\frac{1}{4}}\right], \quad n=1,2,3, \ldots
$$

where $[x]$ denotes the maximal integer that is not larger than $x$.

\section{Main results}

Theorem 3.1 Let E be a real uniformly smooth and strictly convex Banach space with the Kadec-Klee property, let $C$ be a nonempty closed convex subset of $E$, and let $T_{i}: C \rightarrow C, i=$ $1,2, \ldots$, be a countable family of closed and totally quasi- $\phi$-asymptotically nonexpansive mappings with nonnegative real sequences $\left\{v_{n}^{(i)}\right\},\left\{\mu_{n}^{(i)}\right\}$ satisfying $v_{n}^{(i)} \rightarrow 0$ and $\mu_{n}^{(i)} \rightarrow 0$ (as $n \rightarrow \infty$ and for each $i \geq 1)$ and a strictly increasing and continuous function $\zeta: \mathbb{R}^{+} \rightarrow \mathbb{R}^{+}$ satisfying condition (1.1) and each $T_{i}$ is uniformly $L_{i}$-Lipschitz continuous. Let $\left\{\alpha_{n}\right\}$ be a 
sequence in $[0,1]$ with $\alpha_{n} \rightarrow 0$. Let $\left\{x_{n}\right\}$ be the sequence generated by

$$
\left\{\begin{array}{l}
x_{1} \in C ; \quad C_{1}=C, \\
y_{n}=J^{-1}\left[\alpha_{n} J x_{1}+\left(1-\alpha_{n}\right) J T_{i_{n}}^{m_{n}} x_{n}\right], \\
C_{n+1}=\left\{z \in C_{n}: \phi\left(z, y_{n}\right) \leq \alpha_{n} \phi\left(z, x_{1}\right)+\left(1-\alpha_{n}\right) \phi\left(z, x_{n}\right)+\xi_{n}\right\} \\
x_{n+1}=\Pi_{C_{n+1}} x_{1}, \quad \forall n \geq 1,
\end{array}\right.
$$

where $\xi_{n}:=v_{m_{n}}^{\left(i_{n}\right)} \sup _{p \in F} \zeta\left(\phi\left(p, x_{n}\right)\right)+\mu_{m_{n}}^{\left(i_{n}\right)}, \Pi_{C_{n+1}}$ is the generalized projection of E onto $C_{n+1}$, and $i_{n}$ and $m_{n}$ are the solutions to the positive integer equation: $n=i+\frac{(m-1) m}{2}(m \geq i$, $n=1,2, \ldots)$, that is, for each $n \geq 1$, there exist unique $i_{n}$ and $m_{n}$ such that

$$
\begin{array}{ll}
i_{1}=1, & i_{2}=1, \quad i_{3}=2, \quad i_{4}=1, \\
i_{5}=2, & i_{6}=3, \quad i_{7}=1, \quad i_{8}=2, \ldots ; \\
m_{1}=1, & m_{2}=2, \quad m_{3}=2, \quad m_{4}=3, \\
m_{5}=3, & m_{6}=3, \quad m_{7}=4, \quad m_{8}=4, \ldots .
\end{array}
$$

If $F:=\bigcap_{i=1}^{\infty} F\left(T_{i}\right)$ is bounded and $\mu_{1}^{(i)}=0$ for each $i \geq 1$, then $\left\{x_{n}\right\}$ converges strongly to $\Pi_{F} x_{1}$.

Proof We divide the proof into several steps.

(I) $F$ and $C_{n}(\forall n \geq 1)$ both are closed and convex subsets in $C$.

In fact, it follows from Lemma 2.4 that each $F\left(T_{i}\right)$ is a closed and convex subset of $C$, so is $F$. In addition, with $C_{1}(=C)$ being closed and convex, we may assume that $C_{n}$ is closed and convex for some $n \geq 2$. In view of the definition of $\phi$, we have that

$$
C_{n+1}=\{z \in C: \varphi(z) \leq a\} \cap C_{n}
$$

where $\varphi(z)=2 \alpha_{n}\left\langle z, J x_{1}\right\rangle+2\left(1-\alpha_{n}\right)\left\langle z, J x_{n}\right\rangle-2\left\langle z, J y_{n}\right\rangle$ and $a=\alpha_{n}\left\|x_{1}\right\|^{2}+\left(1-\alpha_{n}\right)\left\|x_{n}\right\|^{2}-\left\|y_{n}\right\|^{2}+$ $\xi_{n}$. This shows that $C_{n+1}$ is closed and convex.

(II) $F$ is a subset of $\bigcap_{n=1}^{\infty} C_{n}$.

It is obvious that $F \subset C_{1}$. Suppose that $F \subset C_{n}$ for some $n \geq 2$. Then, for any $p \in F \subset C_{n}$, we have

$$
\begin{aligned}
\phi\left(p, y_{n}\right) & =\phi\left(p, J^{-1}\left[\alpha_{n} J x_{1}+\left(1-\alpha_{n}\right) J T_{i_{n}}^{m_{n}} x_{n}\right]\right) \\
& =\|p\|^{2}-2\left(p, \alpha_{n} J x_{1}+\left(1-\alpha_{n}\right) J T_{i_{n}}^{m_{n}} x_{n}\right\rangle+\left\|\alpha_{n} J x_{1}+\left(1-\alpha_{n}\right) J T_{i_{n}}^{m_{n}} x_{n}\right\|^{2} \\
& \leq\|p\|^{2}-2 \alpha_{n}\left\langle p, J x_{1}\right\rangle-2\left(1-\alpha_{n}\right)\left\langle p, J T_{i_{n}}^{m_{n}} x_{n}\right\rangle+\alpha_{n}\left\|x_{1}\right\|^{2}+\left(1-\alpha_{n}\right)\left\|T_{i_{n}}^{m_{n}} x_{n}\right\|^{2} \\
& =\alpha_{n} \phi\left(p, x_{1}\right)+\left(1-\alpha_{n}\right) \phi\left(p, T_{i_{n}}^{m_{n}} x_{n}\right) \\
& \leq \alpha_{n} \phi\left(p, x_{1}\right)+\left(1-\alpha_{n}\right)\left[\phi\left(p, x_{n}\right)+v_{m_{n}}^{\left(i_{n}\right)} \zeta\left(\phi\left(p, x_{n}\right)\right)+\mu_{m_{n}}^{\left(i_{n}\right)}\right] \\
& \leq \alpha_{n} \phi\left(p, x_{1}\right)+\left(1-\alpha_{n}\right)\left[\phi\left(p, x_{n}\right)+v_{m_{n}}^{\left(i_{n}\right)} \sup _{p \in F} \zeta\left(\phi\left(p, x_{n}\right)\right)+\mu_{m_{n}}^{\left(i_{n}\right)}\right] \\
& =\alpha_{n} \phi\left(p, x_{1}\right)+\left(1-\alpha_{n}\right) \phi\left(p, x_{n}\right)+\xi_{n} .
\end{aligned}
$$

This implies that $p \in C_{n+1}$, and so $F \subset C_{n+1}$. 
(III) $x_{n} \rightarrow x^{*} \in C$ as $n \rightarrow \infty$.

In fact, since $x_{n}=\Pi_{C_{n}} x_{1}$, from Lemma 2.1(2) we have $\left\langle x_{n}-y, J x_{1}-J x_{n}\right\rangle \geq 0, \forall y \in$ $C_{n}$. Again since $F \subset \bigcap_{n=1}^{\infty} C_{n}$, we have $\left\langle x_{n}-p, J x_{1}-J x_{n}\right\rangle \geq 0, \forall p \in F$. It follows from Lemma 2.1(1) that for each $p \in F$ and for each $n \geq 1$,

$$
\phi\left(x_{n}, x_{1}\right)=\phi\left(\Pi_{C_{n}} x_{1}, x_{1}\right) \leq \phi\left(p, x_{1}\right)-\phi\left(p, x_{n}\right) \leq \phi\left(p, x_{1}\right),
$$

which implies that $\left\{\phi\left(x_{n}, x_{1}\right)\right\}$ is bounded, so is $\left\{x_{n}\right\}$. Since for all $n \geq 1, x_{n}=\Pi_{C_{n}} x_{1}$ and $x_{n+1}=\Pi_{C_{n+1}} x_{1} \in C_{n+1} \subset C_{n}$, we have $\phi\left(x_{n}, x_{1}\right) \leq \phi\left(x_{n+1}, x_{1}\right)$. This implies that $\left\{\phi\left(x_{n}, x_{1}\right)\right\}$ is nondecreasing, hence the limit

$$
\lim _{n \rightarrow \infty} \phi\left(x_{n}, x_{1}\right) \text { exists. }
$$

Since $E$ is reflexive, there exists a subsequence $\left\{x_{n_{i}}\right\}$ of $\left\{x_{n}\right\}$ such that $x_{n_{i}} \rightarrow x^{*} \in C$ as $i \rightarrow \infty$. Since $C_{n}$ is closed and convex and $C_{n+1} \subset C_{n}$, this implies that $C_{n}$ is weakly closed and $x^{*} \in C_{n}$ for each $n \geq 1$. In view of $x_{n_{i}}=\Pi_{C_{n_{i}}} x_{1}$, we have

$$
\phi\left(x_{n_{i}}, x_{1}\right) \leq \phi\left(x^{*}, x_{1}\right), \quad \forall i \geq 1
$$

Since the norm $\|\cdot\|$ is weakly lower semi-continuous, we have

$$
\begin{aligned}
\liminf _{i \rightarrow \infty} \phi\left(x_{n_{i}}, x_{1}\right) & =\liminf _{i \rightarrow \infty}\left(\left\|x_{n_{i}}\right\|^{2}-2\left\langle x_{n_{i}}, J x_{1}\right\rangle+\left\|x_{1}\right\|^{2}\right) \geq\left\|x^{*}\right\|^{2}-2\left\langle x^{*}, J x_{1}\right\rangle+\left\|x_{1}\right\|^{2} \\
& =\phi\left(x^{*}, x_{1}\right),
\end{aligned}
$$

and so

$$
\phi\left(x^{*}, x_{1}\right) \leq \liminf _{i \rightarrow \infty} \phi\left(x_{n_{i}}, x_{1}\right) \leq \limsup _{i \rightarrow \infty} \phi\left(x_{n_{i}}, x_{1}\right) \leq \phi\left(x^{*}, x_{1}\right)
$$

This implies that $\lim _{i \rightarrow \infty} \phi\left(x_{n_{i}}, x_{1}\right)=\phi\left(x^{*}, x_{1}\right)$, and so $\left\|x_{n_{i}}\right\| \rightarrow\left\|x^{*}\right\|$ as $i \rightarrow \infty$. Since $x_{n_{i}} \rightarrow x^{*}$, by virtue of the Kadec-Klee property of $E$, we obtain that

$$
\lim _{i \rightarrow \infty} x_{n_{i}}=x^{*}
$$

Since $\left\{\phi\left(x_{n}, x_{1}\right)\right\}$ is convergent, this, together with $\lim _{i \rightarrow \infty} \phi\left(x_{n_{i}}, x_{1}\right)=\phi\left(x^{*}, x_{1}\right)$, shows that $\lim _{n \rightarrow \infty} \phi\left(x_{n}, x_{1}\right)=\phi\left(x^{*}, x_{1}\right)$. If there exists some subsequence $\left\{x_{n_{j}}\right\}$ of $\left\{x_{n}\right\}$ such that $x_{n_{j}} \rightarrow$ $y$ as $j \rightarrow \infty$, then from Lemma 2.1(1) we have that

$$
\begin{aligned}
\phi\left(x^{*}, y\right) & =\lim _{i, j \rightarrow \infty} \phi\left(x_{n_{i}}, x_{n_{j}}\right)=\lim _{i, j \rightarrow \infty} \phi\left(x_{n_{i}}, \Pi_{C_{n_{j}}} x_{1}\right) \\
& \leq \lim _{i, j \rightarrow \infty}\left(\phi\left(x_{n_{i}}, x_{1}\right)-\phi\left(\Pi_{C_{n_{j}}} x_{1}, x_{1}\right)\right) \\
& =\lim _{i, j \rightarrow \infty}\left(\phi\left(x_{n_{i}}, x_{1}\right)-\phi\left(x_{n_{j}}, x_{1}\right)\right) \\
& =\phi\left(x^{*}, x_{1}\right)-\phi\left(x^{*}, x_{1}\right)=0
\end{aligned}
$$

that is, $x^{*}=y$ and so

$$
\lim _{n \rightarrow \infty} x_{n}=x^{*}
$$


(IV) $x^{*}$ is a member of $F$.

Set $\mathcal{K}_{i}=\left\{k \in \mathbb{N}: k=i+\frac{(m-1) m}{2}, m \geq i, m \in \mathbb{N}\right\}$ for each $i \geq 1$. For example, by Lemma 2.5 and the definition of $\mathcal{K}_{1}$, we have $\mathcal{K}_{1}=\{1,2,4,7,11,16, \ldots\}$ and $i_{1}=i_{2}=i_{4}=i_{7}=i_{11}=i_{16}=$ $\cdots=1$. Then we have

$$
\xi_{k}=v_{m_{k}}^{(i)} \sup _{p \in F} \zeta\left(\phi\left(p, x_{k}\right)\right)+\mu_{m_{k}}^{(i)}, \quad \forall k \in \mathcal{K}_{i}
$$

Note that $\left\{m_{k}\right\}_{k \in \mathcal{K}_{i}}=\{i, i+1, i+2, \ldots\}$, i.e., $m_{k} \uparrow \infty$ as $\mathcal{K}_{i} \ni k \rightarrow \infty$. It follows from (3.3) and (3.4) that

$$
\lim _{\mathcal{K}_{i} \ni k \rightarrow \infty} \xi_{k}=0 .
$$

Since $x_{n+1} \in C_{n+1}$, it follows from (3.1), (3.3) and (3.5) that

$$
\phi\left(x_{k+1}, y_{k}\right) \leq \alpha_{k} \phi\left(x_{k+1}, x_{1}\right)+\left(1-\alpha_{k}\right) \phi\left(x_{k+1}, x_{k}\right)+\xi_{k} \rightarrow 0
$$

as $\mathcal{K}_{i} \ni k \rightarrow \infty$. Since $x_{k} \rightarrow x^{*}$ as $\mathcal{K}_{i} \ni k \rightarrow \infty$, it follows from (3.6) and Lemma 2.3 that

$$
\lim _{\mathcal{K}_{i} \ni k \rightarrow \infty} y_{k}=x^{*}
$$

Note that $T_{i_{k}}^{m_{k}}=T_{i}^{m_{k}}$ whenever $k \in \mathcal{K}_{i}$ for each $i \geq 1$. Since $\left\{x_{k}\right\}_{k \in \mathcal{K}_{i}}$ is bounded, so is $\left\{T_{i}^{m_{k}} x_{k}\right\}_{k \in \mathcal{K}_{i}}$. In view of $\alpha_{k} \rightarrow 0$, hence from (3.1) we have that

$$
\lim _{\mathcal{K}_{i} \ni k \rightarrow \infty}\left\|J y_{k}-J T_{i}^{m_{k}}\right\|=\lim _{\mathcal{K}_{i} \ni k \rightarrow \infty} \alpha_{k}\left\|J x_{1}-J T_{i}^{m_{k}}\right\|=0 .
$$

In addition, $J y_{k} \rightarrow J x^{*}$ implies that $\lim _{\mathcal{K}_{i} \ni k \rightarrow \infty} J T_{i}^{m_{k}}=J x^{*}$. Remark 2.2(ii) yields that, as $\mathcal{K}_{i} \ni k \rightarrow \infty$,

$$
T_{i}^{m_{k}} x_{k} \rightarrow x^{*}, \quad \forall i \geq 1 .
$$

Again, since for each $i \geq 1$, as $\mathcal{K}_{i} \ni k \rightarrow \infty$,

$$
\begin{aligned}
\left|\left\|T_{i}^{m_{k}} x_{k}\right\|-\left\|x^{*}\right\|\right| & =\left|\left\|J\left(T_{i}^{m_{k}} x_{k}\right)\right\|-\left\|J x^{*}\right\|\right| \\
& \leq\left\|J\left(T_{i}^{m_{k}} x_{k}\right)-J x^{*}\right\| \rightarrow 0,
\end{aligned}
$$

this, together with (3.10) and the Kadec-Klee property of E, shows that

$$
\lim _{\mathcal{K}_{i} \ni \rightarrow \infty} T_{i}^{m_{k}} x_{k}=x^{*}, \quad \forall i \geq 1
$$

On the other hand, by the assumptions that for each $i \geq 1, T_{i}$ is uniformly $L_{i}$-Lipschitz continuous, and noting that $m_{k+1}-1=m_{k}$ for all $k \in \mathcal{K}_{i}$, we then have

$$
\begin{aligned}
\left\|T_{i}^{m_{k+1}} x_{k}-T_{i}^{m_{k}} x_{k}\right\| \leq & \left\|T_{i}^{m_{k+1}} x_{k}-T_{i}^{m_{k+1}} x_{k+1}\right\|+\left\|T_{i}^{m_{k+1}} x_{k+1}-x_{k+1}\right\| \\
& +\left\|x_{k+1}-x_{k}\right\|+\left\|x_{k}-T_{i}^{m_{k}} x_{k}\right\|
\end{aligned}
$$




$$
\begin{aligned}
\leq & \left(L_{i}+1\right)\left\|x_{k+1}-x_{k}\right\|+\left\|T_{i}^{m_{k+1}} x_{k+1}-x_{k+1}\right\| \\
& +\left\|x_{k}-T_{i}^{m_{k}} x_{k}\right\| .
\end{aligned}
$$

From (3.11) and $x_{k} \rightarrow x^{*}\left(\mathcal{K}_{i} \ni k \rightarrow \infty\right)$, we have that $\lim _{\mathcal{K}_{i} \ni k \rightarrow \infty}\left\|T_{i}^{m_{k+1}} x_{k}-T_{i}^{m_{k}} x_{k}\right\|=0$ and $\lim _{\mathcal{K}_{i} \ni \rightarrow \infty \infty} T_{i}^{m_{k+1}} x_{k}=x^{*}$, i.e., $\lim _{\mathcal{K}_{i} \ni k \rightarrow \infty} T_{i}\left(T_{i}^{m_{k+1}-1} x_{k}\right)=x^{*}$. It then follows that, for each $i \geq 1$,

$$
\lim _{\mathcal{K}_{i} \ni \rightarrow \infty} T_{i}\left(T_{i}^{m_{k}} x_{k}\right)=x^{*}
$$

In view of the closedness of $T_{i}$, it follows from (3.11) that $T_{i} x^{*}=x^{*}$, namely, for each $i \geq 1$, $x^{*} \in F\left(T_{i}\right)$ and hence $x^{*} \in F$.

(V) $x^{*}=\Pi_{F} x_{1}$, and so $x_{n} \rightarrow \Pi_{F} x_{1}$ as $n \rightarrow \infty$.

Put $u=\Pi_{F} x_{1}$. Since $u \in F \subset C_{n}$ and $x_{n}=\Pi_{C_{n}} x_{1}$, we have $\phi\left(x_{n}, x_{1}\right) \leq \phi\left(u, x_{1}\right), \forall n \geq 1$.

Then

$$
\phi\left(x^{*}, x_{1}\right)=\lim _{n \rightarrow \infty} \phi\left(x_{n}, x_{1}\right) \leq \phi\left(u, x_{1}\right),
$$

which implies that $x^{*}=u$ since $u=\Pi_{F} x_{1}$, and hence $x_{n} \rightarrow x^{*}=\Pi_{F} x_{1}$. This completes the proof.

Remark 3.2 Note that algorithm (3.1) just depends on the projection onto a single closed and convex set for each fixed $n$. An example [9] of how to compute such a projection is given as follows.

Dykstra's algorithm Let $\Omega_{1}, \Omega_{2}, \ldots, \Omega_{p}$ be closed and convex subsets of $\mathbb{R}^{n}$. For any $i=$ $1,2, \ldots, p$ and $x^{0} \in \mathbb{R}^{n}$, the sequences $\left\{x_{i}^{k}\right\}$ are defined by the following recursive formulae:

$$
\left\{\begin{array}{l}
x_{0}^{k}=x_{p}^{k-1}, \\
x_{i}^{k}=P_{\Omega_{i}}\left(x_{i-1}^{k}-y_{i}^{k-1}\right), \quad i=1,2, \ldots, p, \\
y_{i}^{k}=x_{i}^{k}-\left(x_{i-1}^{k}-y_{i}^{k-1}\right), \quad i=1,2, \ldots, p,
\end{array}\right.
$$

for $k=1,2, \ldots$ with initial values $x_{p}^{0}=x^{0}$ and $y_{i}^{0}=0$ for $i=1,2, \ldots, p$. If $\Omega:=\bigcap_{i=1}^{p} \Omega_{i} \neq \emptyset$, then $\left\{x_{i}^{k}\right\}$ converges to $x^{*}=P_{\Omega}\left(x^{0}\right)$, where $P_{\Omega}(x):=\arg \inf _{y \in \Omega}\|y-x\|^{2}, \forall x \in \mathbb{R}^{n}$.

We now give a nontrivial example of the calculation of common fixed points for specific mappings.

Example 3.3 Let $E=\mathbb{R}^{1}$ with the standard norm $\|\cdot\|=|\cdot|$ and $C=[0,1]$. Let $\left\{T_{i}\right\}: C \rightarrow$ $C$ be a sequence of nonexpansive mappings defined by $T_{i} x=\frac{x^{i}}{i}$. Consider the following iteration sequence generated by

$$
\left\{\begin{array}{l}
x_{1} \in C ; \quad C_{1}=C, \\
y_{n}=J^{-1}\left[\alpha_{n} J x_{n}+\left(1-\alpha_{n}\right) J z_{n}\right], \\
z_{n}=J^{-1}\left[\beta_{n} J x_{n}+\left(1-\beta_{n}\right) J T_{i_{n}} x_{n}\right], \\
C_{n+1}=\left\{v \in C_{n}: \phi\left(v, y_{n}\right) \leq \phi\left(v, x_{n}\right)\right\}, \\
x_{n+1}=\Pi_{C_{n+1}} x_{1}, \quad \forall n \geq 1,
\end{array}\right.
$$


where $\left\{\alpha_{n}\right\}=\left\{\frac{2}{3}-\frac{1}{4 n}\right\},\left\{\beta_{n}\right\}=\left\{\frac{4}{5}-\frac{1}{2 n}\right\}$ and $\Pi_{C_{n+1}}(x):=\arg \inf _{y \in C_{n+1}}|y-x|$. Note that $J=I$ and $\phi(x, y)=|x-y|^{2}$ for all $x, y \in E$ since $E$ is a Hilbert space. Moreover, it is not difficult to obtain that $C_{n+1}=\left[0, \frac{x_{n}+y_{n}}{2}\right]$ for all $n \geq 1$. Then (3.16) is reduced to

$$
\left\{\begin{array}{l}
x_{1} \in C ; \quad C_{1}=C \\
y_{n}=\left(\frac{2}{3}-\frac{1}{4 n}\right) x_{n}+\left(\frac{1}{3}+\frac{1}{4 n}\right) z_{n}, \\
z_{n}=\left(\frac{4}{5}-\frac{1}{2 n}\right) x_{n}+\left(\frac{1}{5}+\frac{1}{2 n}\right) T_{i_{n}} x_{n}, \\
C_{n+1}=\left\{v \in C_{n}:\left|v-y_{n}\right| \leq\left|v-x_{n}\right|\right\}, \\
x_{n+1}=\frac{x_{n}+y_{n}}{2}, \quad \forall n \geq 1,
\end{array}\right.
$$

where $i_{n}$ is the solution to the positive integer equation: $n=i+\frac{(m-1) m}{2}(m \geq i, n=1,2, \ldots)$. It is clear that $\left\{T_{i}\right\}$ is a sequence of closed and totally quasi- $\phi$-asymptotically nonexpansive mappings with a common fixed point zero. It then can be shown by a similar way of Theorem 3.1 that $\left\{x_{n}\right\}$ converges strongly to zero. The numerical experiment outcome obtained by using MATLAB 7.10.0.499 shows that as $x_{1}=1$, the computations of $x_{100}, x_{200}, x_{300}$ and $x_{400}$ are $0.025141746,0.00078472044,0.000025282198$ and 0.00000082531714 , respectively. This example illustrates the effectiveness of the introduced algorithm for countable families of totally quasi- $\phi$-asymptotically nonexpansive mappings.

\section{Applications}

The so-called convex feasibility problem for a family of mappings $\left\{T_{i}\right\}_{i=1}^{\infty}$ is to find a point in the nonempty intersection $\bigcap_{i=1}^{\infty} F\left(T_{i}\right)$, which exactly illustrates the importance of finding common fixed points of infinite families. The following example also clarifies the same thing.

Example 4.1 Let $E$ be a smooth, strictly convex and reflexive Banach space, let $C$ be a nonempty and closed convex subset of $E$, and let $\left\{f_{i}\right\}_{i=1}^{\infty}: C \times C \rightarrow \mathbb{R}$ be a sequence of bifunctions satisfying the conditions: for each $i \geq 1$,

$\left(\mathrm{A}_{1}\right) f_{i}(x, x)=0$;

$\left(\mathrm{A}_{2}\right) f_{i}$ is monotone, i.e., $f_{i}(x, y)+f_{i}(y, x) \leq 0$;

$\left(\mathrm{A}_{3}\right) \lim \sup _{t \downarrow 0} f_{i}(x+t(z-x), y) \leq f_{i}(x, y)$;

$\left(\mathrm{A}_{4}\right)$ the mapping $y \mapsto f_{i}(x, y)$ is convex and lower semicontinuous.

A system of equilibrium problems for $\left\{f_{i}\right\}_{i=1}^{\infty}$ is to find an $x^{*} \in C$ such that

$$
f_{i}\left(x^{*}, y\right) \geq 0, \quad \forall y \in C, i \geq 1,
$$

whose set of common solutions is denoted by $E P:=\bigcap_{i=1}^{\infty} E P\left(f_{i}\right)$, where $E P\left(f_{i}\right)$ denotes the set of solutions to the equilibrium problem for $f_{i}(i=1,2, \ldots)$. It is shown in [10, Theorem 4.3] that such a system of problems can be reduced to the approximation of some fixed point of a sequence of nonlinear mappings. 


\section{Acknowledgements}

This work is supported by the National Natural Science Foundation of China (Grant No. 11061037).

Received: 25 April 2013 Accepted: 19 July 2013 Published: 5 August 2013

\section{References}

1. Chang, S-s, Lee, HWJ, Chan, CK, Zhang, WB: A modified Halpern-type iterative algorithm for totally quasi- $\phi$-asymptotically nonexpansive mappings with applications. Appl. Math. Comput. 218(11), 6489-6497 (2012)

2. Qin, $\mathrm{XL}, \mathrm{Su}, \mathrm{YF}$ : Strong convergence theorems for relatively nonexpansive mappings in a Banach space. Nonlinear Anal. 67, 1958-1965 (2007)

3. Qin, XL, Cho, YJ, Kang, SM, Zhou, HY: Convergence of a modified Halpern-type iterative algorithm for quasi- $\boldsymbol{\phi}$-nonexpansive mappings. Appl. Math. Lett. 22, 1051-1055 (2009)

4. Wang, ZM, Su, YF, Wang, DX, Dong, YC: A modified Halpern-type iteration algorithm for a family of hemi-relative nonexpansive mappings and systems of equilibrium problems in Banach spaces. J. Comput. Appl. Math. 235 2364-2371 (2011)

5. Martinez-Yanes, C, Xu, HK: Strong convergence of the CQ method for fixed point iteration processes. Nonlinear Anal. 64, 2400-2411 (2006)

6. Alber, Yl: Metric and generalized projection operators in Banach spaces: properties and applications. In: Kartosator, AG (ed.) Theory and Applications of Nonlinear Operators of Accretive and Monotone Type, pp. 15-50. Dekker, New York (1996)

7. Cioranescu, I: Geometry of Banach Spaces, Duality Mappings and Nonlinear Problems. Kluwer Academic, Dordrecht (1990)

8. Deng, W-Q, Bai, P: An implicit iteration process for common fixed points of two infinite families of asymptotically nonexpansive mappings in Banach spaces. J. Appl. Math. 2013, Article ID 602582 (2013)

9. Boyle, JP, Dykstra, RL: A method for finding projections onto the intersections of convex sets in Hilbert spaces. In: Advances in Order Restricted Statistical Inference. Lecture Notes in Statistics, vol. 37, pp. 28-47 (1986)

10. Deng, W-Q: A new approach to the approximation of common fixed points of an infinite family of relatively quasinonexpansive mappings with applications. Abstr. Appl. Anal. 2012, Article ID 437430 (2012)

doi:10.1186/1029-242X-2013-367

Cite this article as: Deng: Relaxed Halpern-type iteration method for countable families of totally quasi- $\phi$-asymptotically nonexpansive mappings. Journal of Inequalities and Applications 20132013.367.

\section{Submit your manuscript to a SpringerOpen ${ }^{\circ}$ journal and benefit from:}

- Convenient online submission

- Rigorous peer review

- Immediate publication on acceptance

- Open access: articles freely available online

- High visibility within the field

- Retaining the copyright to your article 